EPJ Web of Conferences 49, 08002 (2013)

DOI: $10.1051 /$ epjconf/20134908002

(C) Owned by the authors, published by EDP Sciences, 2013

\title{
Diboson production
}

\author{
D.L.Evans ${ }^{1, a}$ \\ On behalf of the ATLAS, CMS, CDF and D $\varnothing$ Collaborations \\ ${ }^{1}$ University of California, San Diego (UCSD) \\ 9500 Gilman Drive \#0319, La Jolla, CA 92093-0319 \\ USA
}

\begin{abstract}
Measurements of diboson production cross sections in pp collisions at the LHC at a centre of mass energy $\sqrt{s}=7$ and $8 \mathrm{TeV}$, and in $\mathrm{p} \overline{\mathrm{p}}$ collisions at the Tevatron at $\sqrt{s}=1.96 \mathrm{TeV}$ are reviewed and compared with standard model predictions. Limits on charged and neutral anomalous triple gauge couplings extracted from the selected diboson event samples are also compared.
\end{abstract}

\section{Introduction}

The measurement of diboson production provides an important test of the standard model (SM), with any significant deviation from the measured cross sections giving an indication of new physics. In the SM, diboson production proceeds principally through the quark anti-quark annihilation process, shown in Figure 1. Triple gauge boson couplings (TGC) in the s-channel production mode are sensitive to new physics at a higher mass scale, which could change both the production rate and kinematics. In addition, measurements of diboson production are essential for an accurate estimate of irreducible backgrounds to the newly discovered resonance $[1,2]$ in the diboson decay modes.
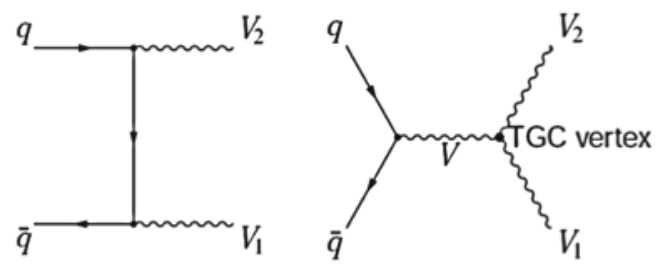

Figure 1. Diboson production in the t-channel (left) and schannel (right).

Several recent results have been presented at this conference, using $\mathrm{p} \overline{\mathrm{p}}$ collision data from the Tevatron at a centre of mass energy $\sqrt{s}=1.96 \mathrm{TeV}$, and in pp collisions at $\sqrt{s}=7$ and $8 \mathrm{TeV}$ at the LHC. Cross section measurements are described in Section 2 and limits on anomalous triple gauge couplings are compared in Section 3.

\footnotetext{
ae-mail: d.evans@cern.ch
}

\section{Cross Section Measurements}

\section{1 $\mathrm{W} \gamma$ and $\mathrm{Z} \gamma$ final states}

From the various diboson processes that can be produced at hadron colliders, the $\mathrm{W} \gamma$ and $\mathrm{Z} \gamma$ final states have the highest yields, next to $\gamma \gamma$ production. The presence of either a $\mathrm{W}$ or $\mathrm{Z}$ boson improves the suppression of background processes, thus allowing stringent checks of the electroweak sector to be performed in these final sates.

Events are selected in the $\mathrm{W} \gamma$ final state by requiring the presence of an electron or muon $(\ell)$, and missing transverse energy $E_{\mathrm{T}}^{\text {miss }}$ from the undetected neutrino, in addition to a photon. To suppress the contribution from final state radiation photons, measurements are reported for $\Delta R(\ell, \gamma)=\sqrt{d \eta^{2}+d \phi^{2}}>0.7$. The dominant background comes from $\mathrm{W}+$ jets events, where the jet is misidentified as a photon.

The ATLAS and CMS collaborations have performed measurements of $\sigma(\mathrm{pp} \rightarrow \mathrm{W} \gamma)$ at a centre of mass energy of $\sqrt{s}=7 \mathrm{TeV}$, by using datasets corresponding to an integrated luminosity of $1.02 \mathrm{fb}^{-1}$ and $5 \mathrm{fb}^{-1}$ respectively. The ATLAS measurement of the inclusive cross section is $4.60 \pm 0.11$ (stat.) \pm 0.64 (syst.) pb for photon $p_{\mathrm{T}}>15 \mathrm{GeV}$ in an extended fiducial region, corresponding to the NLO SM prediction of $3.70 \pm 0.28 \mathrm{pb}$ [3]. The extended fiducial region requires the lepton and neutrino transverse momentum, $p_{\mathrm{T}}$, to be above $25 \mathrm{GeV}$, and the lepton to be within pseudorapidity, $|\eta|<2.47$, to emulate the experimental selection. The CMS collaboration has presented new results for this conference, measuring the inclusive cross section to be $37.0 \pm 0.8$ (stat.) \pm 4.0 (syst.) \pm 0.8 (lumi.) pb, to be compared with the NLO SM prediction of $31.81 \pm 1.8 \mathrm{pb}$ for photon $p_{\mathrm{T}}$ above $15 \mathrm{GeV}[4]$. The CDF and D $\varnothing$ collaborations have performed measurements of $\sigma(\mathrm{p} \overline{\mathrm{p}} \rightarrow \mathrm{W} \gamma)$ by analysing datasets corresponding to an integrated luminosity of $1 \mathrm{fb}^{-1}$ and $4.2 \mathrm{fb}^{-1}$ respectively. The CDF 
collaboration measured $7.36 \pm 0.35$ (stat.) \pm 0.75 (syst.) \pm 0.43 (lumi.) pb to be compared with the NLO SM prediction for photons with $p_{\mathrm{T}}$ above $15 \mathrm{GeV}$ and $|\eta|<2.0$ of $8.2 \pm 0.6 \mathrm{pb}$ [5]. The $\mathrm{D} \varnothing$ collaboration measured $7.6 \pm 0.6$ (stat.) \pm 0.6 (syst.) pb to be compared with the NLO SM prediction of $7.6 \pm 0.2 \mathrm{pb}$ for photons with $p_{\mathrm{T}}$ above $15 \mathrm{GeV}$ [6]. These results are compared in Figure 2.

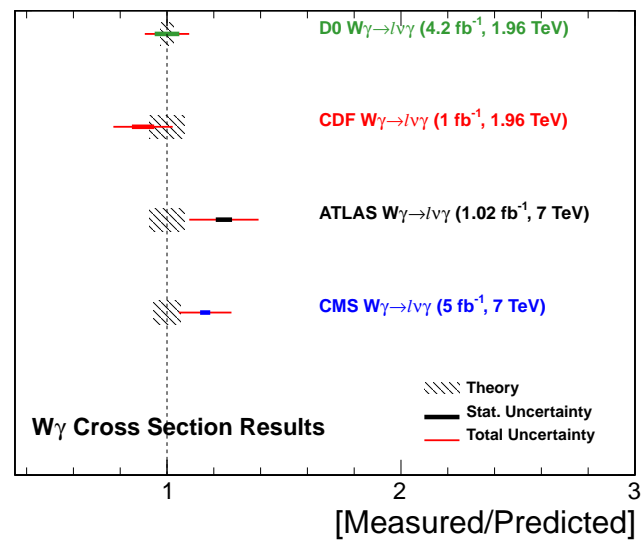

Figure 2. Summary of $\mathrm{W} \gamma$ cross section measurements compared to standard model predictions.

In the $\mathrm{Z} \gamma$ analyses, events are selected by requiring a same flavor, opposite sign electron or muon pair $(\ell \ell)$ with an invariant mass $M_{\ell \ell}$ close to the $\mathrm{Z}$ boson mass, in addition to a photon. The ATLAS collaboration has measured the inclusive $\sigma(\mathrm{pp} \rightarrow \mathrm{Z} \gamma)$ cross section of $1.29 \pm 0.05$ (stat.) \pm 0.15 (syst.) pb to be compared with the NLO SM prediction of $1.23 \pm 0.06 \mathrm{pb}$ in the extended fiducial region [3]. The CMS collaboration has measured an inclusive cross section of $5.33 \pm 0.08$ (stat.) \pm 0.25 (syst.) \pm 0.12 (lumi.) pb to be compared with the NLO SM prediction of $5.45 \pm 0.27 \mathrm{pb}$ [4] for photon $p_{\mathrm{T}}>15 \mathrm{GeV}$ and $M_{\ell \ell}>50 \mathrm{GeV}$. The CDF collaboration has measured the inclusive $\sigma(\mathrm{p} \overline{\mathrm{p}} \rightarrow \mathrm{Z} \gamma)$ cross section of $4.6 \pm 0.2$ (stat.) \pm 0.3 (syst.) \pm 0.4 (lumi.) pb to be compared with the NLO SM prediction of $4.5 \pm 0.4 \mathrm{pb}$ in the fiducial region with photon $p_{\mathrm{T}}$ above $7 \mathrm{GeV}$ and a dilepton invariant mass of at least $40 \mathrm{GeV}$ [7]. The $\mathrm{D} \varnothing$ collaboration measured $288 \pm 15$ (stat.) \pm 11 (syst.) fb to be compared to the theoretical prediction of $294 \pm 10$ (PDF) ${ }_{-2}^{+1}$ (scale) fb, with a requirement of $M_{\ell \ell \gamma}>110 \mathrm{GeV}$ [8]. These results are compared in Figure 3.

The first measurement of $\mathrm{Z} \gamma$ production in the $v v \gamma$ final state in pp collisions at $\sqrt{s}=7 \mathrm{TeV}$ has been presented by the CMS collaboration, using a dataset corresponding to an integrated luminosity of $5 \mathrm{fb}^{-1}$. This measurement is challenging because of large backgrounds from jets misidentified as photons, and instrumental sources such as beam-gas interactions. The $\mathrm{Z} \gamma$ production cross section is measured to be $21.3 \pm 4.2$ (stat.) \pm 4.3 (syst.) \pm 0.5 (lumi.) $\mathrm{fb}$, where the photon is required to have $|\eta|<1.4$ and $E_{\mathrm{T}}>145 \mathrm{GeV}$, in agreement with the SM prediction of $21.9 \pm 1.1 \mathrm{fb}$ [9]. A previous measurement in this final

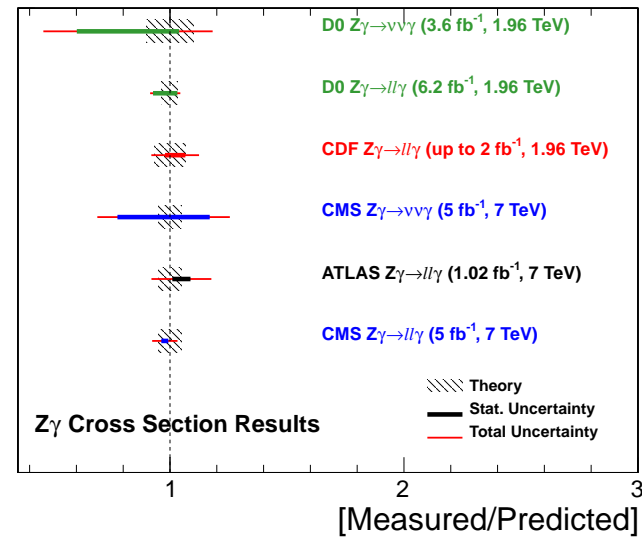

Figure 3. Summary of $Z \gamma$ cross section measurements compared to standard model predictions.

state at $\sqrt{s}=1.96 \mathrm{TeV}$ in $\mathrm{p} \overline{\mathrm{p}}$ collisions was also presented. The $\mathrm{D} \varnothing$ collaboration measured a cross section of $32 \pm 9$ (stat. + syst. \pm 2 (lumi.) fb to be compared with the NLO SM prediction of $39 \pm 4$ fb for photon $p_{\mathrm{T}}$ above $90 \mathrm{GeV}[10]$. These results are also compared in Figure 3.

\subsection{WW and WZ final states}

The ATLAS and CMS collaborations have measured the WW production cross section in the fully leptonic $(\ell v \ell v)$ final state at $\sqrt{s}=7 \mathrm{TeV}$, by using datasets corresponding to $4.6 \mathrm{fb}^{-1}$ and $4.92 \mathrm{fb}^{-1}$ respectively. CMS has also measured the production cross section in this final state at $\sqrt{s}=8 \mathrm{TeV}$ corresponding to an integrated luminosity of $3.54 \mathrm{fb}^{-1}$. The $\mathrm{SM}$ background sources to the $\mathrm{W}^{+} \mathrm{W}^{-}$ event sample in the $\ell \nu \ell v$ final state include $\mathrm{W} \gamma^{(*)}$, topquark ( $\mathrm{t} \overline{\mathrm{t}}$ and $\mathrm{tW}), \mathrm{Z} / \gamma^{*} \rightarrow \ell^{+} \ell^{-}$, and diboson (WZ and $\mathrm{ZZ}$ ) production, as well as $\mathrm{W}+$ jets and QCD multijet events, where at least one of the jets is misidentified as a lepton. The $\mathrm{Z} / \gamma^{*} \rightarrow \ell^{+} \ell^{-}$background is suppressed by requiring large $E_{\mathrm{T}}^{\text {miss }}$ and dilepton $p_{\mathrm{T}}$, and the $\mathrm{W}+$ jets background is reduced by using stringent lepton identification and isolation requirements. The jet multiplicity distribution in the ATLAS event sample with two leptons of different flavor is shown in Figure 4. To minimise the contribution from the top-quark background, events containing jets are rejected. This leads to a significant theoretical uncertainty in the jet veto efficiency.

The ATLAS and CMS measurements of $\sigma(\mathrm{pp} \rightarrow \mathrm{WW})$ at $\sqrt{s}=7 \mathrm{TeV}$ are $51.9 \pm 2.0$ (stat.) \pm 3.9 (syst.) \pm 2.0 (lumi.) pb and $52.4 \pm 2.0$ (stat.) \pm 4.5 (syst.) \pm 1.2 (lumi.) pb, respectively [11] [12]. Both measurements are in agreement with the NLO SM prediction of $47.0 \mathrm{pb}$ [13]. The CMS measurement at $\sqrt{s}=8 \mathrm{TeV}$ is $69.9 \pm 2.8$ (stat.) \pm 5.6 (syst.) \pm 3.1 (lumi.) pb, corresponding to the NLO SM prediction of $57.3_{-1.6}^{+2.4} \mathrm{pb}$ [14]. The difference between the measured and theoretical value is $(22 \pm 13) \%$ of the theoretical value.

The ATLAS, CMS, and D $\varnothing$ collaborations have also measured the total cross section of $\mathrm{WW}+\mathrm{WZ}$ produc- 


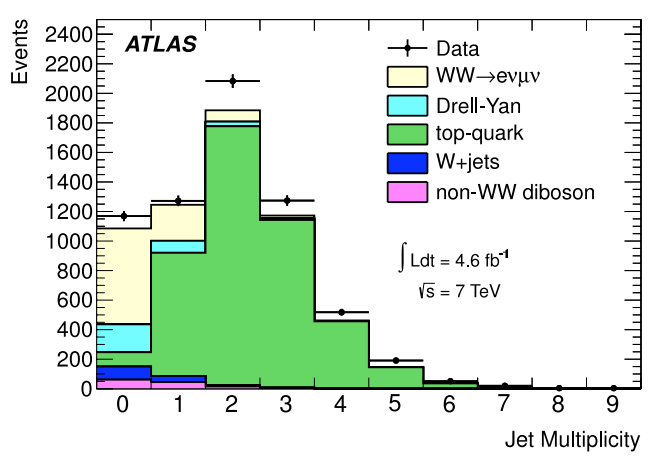

Figure 4. Comparison between the ATLAS WW $\rightarrow e v \mu v$ data and simulation for the jet multiplicity before the jet veto requirement.

tion by selecting events in the semi leptonic $(\ell v j j)$ decay mode where one $\mathrm{W}$ boson decays leptonically and the other boson (W or Z) decays hadronically. This decay mode has a higher branching fraction compared to the fully leptonic mode, at the expense of introducing a larger background from $\mathrm{W}+$ jets events. The ATLAS and CMS measurements used datasets corresponding to an integrated luminosity of $4.7 \mathrm{fb}^{-1}$ and $5 \mathrm{fb}^{-1}$ at $\sqrt{s}=7 \mathrm{TeV}$, and the D $\varnothing$ measurement used a dataset corresponding to $4.3 \mathrm{fb}^{-1}$. ATLAS measured $\sigma(\mathrm{pp} \rightarrow(\mathrm{WW}+\mathrm{WZ}))$ to be $72 \pm 9$ (stat.) \pm 15 (syst.) \pm 13 (MC stat.) pb using a dataset corresponding to $4.7 \mathrm{fb}^{-1}$, consistent with the SM expectation of $63.4 \pm 2.6 \mathrm{pb}$. CMS measured $68.9 \pm 8.7$ (stat.) \pm 9.7 (syst.) \pm 1.5 (lumi.) pb [15]. The $\mathrm{D} \emptyset$ collaboration measured $\sigma(\mathrm{p} \overline{\mathrm{p}} \rightarrow(\mathrm{WW}+\mathrm{WZ}))$ to be $19.6_{-3.0}^{+3.2} \mathrm{pb}$, compared with the NLO SM prediction of $15.2 \pm 0.85 \mathrm{pb}[16]$.

Measurements of WZ production in the fully leptonic $(\ell v \ell \ell)$ final state have high purity after requiring three high $p_{\mathrm{T}}$ leptons, two of which are same flavor and have an invariant mass close to the $\mathrm{Z}$ boson, and large $E_{\mathrm{T}}^{\text {miss }}$ from the $\mathrm{W}$ decay. Because the branching ratio to this final state is smaller, these measurements are dominated by the statistical uncertainty in the selected event sample. The ATLAS and CMS collaborations have performed measurements of $\sigma(\mathrm{pp} \rightarrow \mathrm{WZ})$ at $\sqrt{s}=7 \mathrm{TeV}$ by analysing datasets corresponding to an integrated luminosity of $4.6 \mathrm{fb}^{-1}$ and $1 \mathrm{fb}^{-1}$ respectively. ATLAS measured a total cross section of $19.0_{-1.3}^{+1.4}$ (stat.) \pm 0.9 (syst.) \pm 0.4 (lumi.) pb compared with the NLO SM prediction of $17.6_{-1.0}^{+1.1} \mathrm{pb}$ for the dilepton invariant mass range $66<M_{\ell \ell}<116 \mathrm{GeV}$ for $\mathrm{Z} \rightarrow \ell \ell$ [17]. CMS measured a total cross section of $17.0 \pm 2.4$ (stat.) \pm 1.1 (syst.) \pm 1.0 (lumi.) pb, which agrees well with an NLO SM prediction of $19.790 \pm$ $0.088 \mathrm{pb}$ [18]. The CDF and DØ measurements of $\sigma(\mathrm{p} \overline{\mathrm{p}} \rightarrow \mathrm{WZ})$ are $3.94_{-0.53}^{+0.60}$ (stat. $)_{-0.46}^{+0.59}$ (syst.) pb, and $4.50 \pm 0.61$ (stat. $)_{-0.25}^{+0.16}$ (syst.) pb for $60<M_{\ell \ell}<120 \mathrm{GeV}$, in datasets corresponding to $7.2 \mathrm{fb}^{-1}$ and $8.6 \mathrm{fb}^{-1}$ respectively [19] [20]. The CDF measurement is in good agreement with the NLO SM prediction of $3.50 \pm 0.21 \mathrm{pb}$, and the $\mathrm{D} \varnothing$ measurement is slightly larger than, but still consistent with the NLO SM prediction of $3.21 \pm 0.19 \mathrm{pb}$.

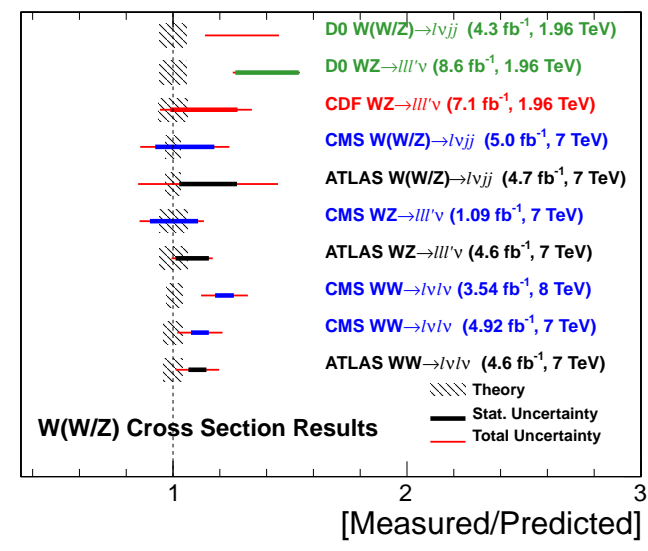

Figure 5. Summary of WW and WZ cross section measurements compared to standard model predictions.

These results are displayed graphically, compared with standard model predictions in Figure 5.

\section{3 $\mathrm{ZZ}$ final state}

Measurements of $\mathrm{ZZ}$ production have been performed in the clean, but statistically limited four-lepton $\left(\ell^{+} \ell^{-} \ell^{+} \ell^{-}\right)$ final state by the ATLAS, CMS, CDF and D $\varnothing$ collaborations. Because the branching ratio for this final state is small, the precision of the measurements can be improved by combining with higher branching ratio final states in which one of the $\mathrm{Z}$ bosons decays either invisibly or to $\tau$ leptons.

The ATLAS collaboration measured $\sigma(\mathrm{pp} \rightarrow \mathrm{ZZ})$ $6.7 \pm 0.7$ (stat. $)_{-0.3}^{+0.4}$ (syst.) \pm 0.3 (lumi.) pb, by combining the four-lepton results with a measurement where of the $\mathrm{Z}$ bosons decays invisibly in the dilepton $+E_{\mathrm{T}}^{\mathrm{miss}}$ final state, both using a dataset corresponding to $4.6 \mathrm{fb}^{-1}$ at $\sqrt{s}=7 \mathrm{TeV}$. This result is consistent within uncertainties with the NLO SM prediction of $5.89_{-0.18}^{+0.22} \mathrm{pb}$ in the fiducial region $66<M_{\ell \ell}<106 \mathrm{GeV}$ [21]. The CMS collaboration measured $6.24_{-0.80}^{+0.86}$ (stat.) ${ }_{-0.32}^{+0.41}$ (syst.) \pm 0.14 (lumi.) pb in the fiducial region $60<M_{\ell \ell}<120 \mathrm{GeV}$, including both four-lepton events and those where the second $\mathrm{Z}$ boson decays to $\tau$ leptons. This result is consistent with the NLO SM prediction of $6.3 \pm 0.4 \mathrm{pb}$ [22].

ATLAS measured $\quad 9.3_{-1.0}^{+1.1} \quad$ (stat. $)_{-0.3}^{+0.4} \quad$ (syst.) $\quad \pm$ 0.3 (lumi.) $\mathrm{pb}$ in the four-lepton final state by using a dataset corresponding to $5.8 \mathrm{fb}^{-1}$ at $\sqrt{s}=8 \mathrm{TeV}$. This measurement is in agreement with the NLO SM prediction of $7.4 \pm 0.4 \mathrm{pb}$ in the fiducial region where the leading lepton has $p_{\mathrm{T}}>25 \mathrm{GeV}$, the invariant mass of both opposite-sign same-flavor lepton pairs is between $66<M_{\ell \ell}<106 \mathrm{GeV}$ and electrons (muons) are required to be in $|\eta|<2.47$ (2.50) [23]. CMS has measured $8.4 \pm 1.0$ (stat.) \pm 0.7 (syst.) \pm 0.4 (lumi.) pb using a dataset corresponding to $5.26 \mathrm{fb}^{-1}$, including both four-lepton and two lepton two $\tau$ events, to be compared with the the NLO SM prediction of $7.7 \pm 0.4 \mathrm{pb}$ in the fiducial region $60<M_{\ell \ell}<120 \mathrm{GeV}$ [14]. 


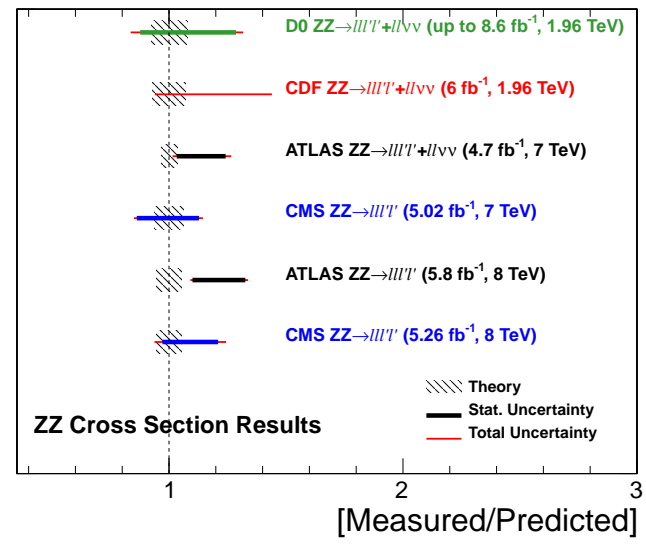

Figure 6. Summary of ZZ cross section measurements compared to standard model predictions.

The CDF and D $\varnothing$ collaborations measured $\sigma(\mathrm{p} \overline{\mathrm{p}} \rightarrow \mathrm{ZZ})$ by using datasets corresponding to $6 \mathrm{fb}^{-1}$ and up to $8.6 \mathrm{fb}^{-1}$ respectively. Both measurements are combinations of results in the four-lepton and two lepton $+E_{\mathrm{T}}^{\mathrm{miss}}$ final states. CDF measured $1.64_{-0.38}^{+0.44}$ (stat + syst) $\mathrm{pb}$, which is consistent with the NLO SM prediction of $1.4 \pm 0.1 \mathrm{pb}$ [24]. Equivalently, D $\varnothing$ measured $1.44_{-0.28}^{+0.31}$ (stat.) $\pm_{-0.19}^{+0.17}$ (syst.) pb [20].

The $\mathrm{ZZ}$ cross section measurements are summarised in comparison with the relevant theoretical predictions in Figure 6.

The CMS collaboration has performed the first observation of $\mathrm{Z} \rightarrow 4 \ell$ decays in pp collisions at $\sqrt{s}=7 \mathrm{TeV}$, by analysing a dataset corresponding to an integrated luminosity of $5.02 \pm 0.11 \mathrm{fb}^{-1}$. The signal, shown in Figure 7, was observed with a statistical significance of $9.7 \sigma$ [25]. The measured branching fraction is $\mathcal{B}(Z \rightarrow 4 \ell)=$ $\left(4.2_{-0.8}^{+0.9}\right.$ (stat.) \pm 0.2 (syst. $\left.)\right) \times 10^{-6}$, in agreement with the SM prediction of $4.45 \times 10^{-6}$. The measured cross section times branching fraction is $\sigma(\mathrm{pp} \rightarrow \mathrm{Z}) \mathcal{B}(\mathrm{Z} \rightarrow 4 \ell)=$ $112_{-20}^{+23}$ (stat. $)_{-5}^{+7}$ (syst. $)_{-2}^{+3}$ (lumi.), which is also in agreement with the SM prediction of $120 \mathrm{fb}^{-1}$. These measurements were performed with the four-lepton invariant mass restricted to $80<m_{4 \ell}<100 \mathrm{GeV}$ and the two-lepton invariant mass restricted to $m_{\ell \ell}>4 \mathrm{GeV}$ for all pairs of leptons. In addition, because the $\mathrm{Z} \rightarrow 4 \ell$ gives a narrow resonant peak in the four-lepton invariant mass distribution, this channel can be used to calibrate the four-lepton mass scale and low $p_{\mathrm{T}}$ lepton identification efficiencies for Higgs boson measurements in the $\mathrm{ZZ} \rightarrow 4 \ell$ decay mode.

\section{Limits on anomalous triple gauge couplings}

Diboson production is sensitive to triple gauge couplings in the s-channel production modes. Deviations of the triple gauge couplings from the SM values are referred to as anomalous triple gauge couplings (aTGC), which are parameterised by adding terms to the SM Lagrangian. The presence of aTGC will modify both the production rate

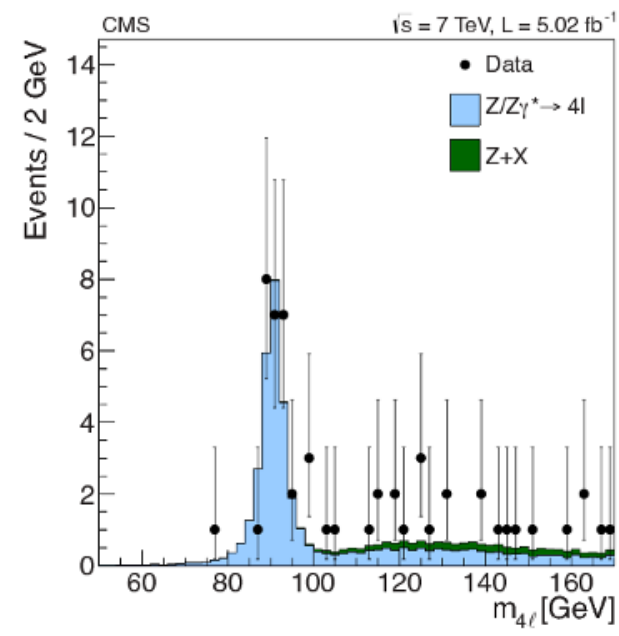

Figure 7. Four-lepton invariant mass distribution for events passing all selection requirements except that on $m_{4 \ell}$. The data are shown by points. The filled histograms represent SM expectations for $\mathrm{pp} \rightarrow \mathrm{Z} / \mathrm{Z} \gamma^{\star} \rightarrow 4 \ell$ and for reducible backgrounds. The three final states, $4 \mathrm{e}, 4 \mu$, and $2 \mathrm{e} 2 \mu$ are combined.

and kinematics from SM expectations. To prevent the total production cross section violating unitarity, a form factor is introduced to turn off the effect of each parameter above a cut off scale $\Lambda$. As the experimental sensitivity to aTGC increases, so does the scale at which unitarity violation would occur for permitted aTGC values.

Experimental measurements have been made by the ATLAS, CMS, CDF and DØ collaborations, leading to limits on charged and neutral aTGC. These limits are now summarised and compared.

\section{Charged couplings}

For the WW $\gamma$ vertex, the aTGC parameters are chosen to be $\lambda_{\gamma}$ and $\Delta \kappa_{\gamma}=\kappa_{\gamma}-1$, which are zero in the SM [26]. By using the photon $p_{\mathrm{T}}$ spectrum in the $\mathrm{W} \gamma$ final state, CMS, ATLAS, and D $\varnothing$ have set limits on aTGC in the $\mathrm{WW} \gamma$ vertex [4], [3] [6]. ATLAS and CMS have set limits by using the leading lepton $p_{\mathrm{T}}$ in the fully leptonic WW final state, which is also sensitive to the WW $\gamma$ vertex [11] [27]. By exploiting the higher branching ratio semi leptonic final state, CMS has set stringent limits from the dijet $p_{\mathrm{T}}$ spectrum [15]. These results are summarised graphically in Figure 8. The LHC measurements are now almost the most stringent, with LEP still providing more stringent limits on $\Delta \kappa_{\gamma}$ [28].

The aTGC parameters for the WW $\gamma$ and WWZ vertices are chosen to be $\Delta g_{1}^{Z}=g_{1}^{Z}-1, \Delta \kappa_{Z}=\kappa_{Z}-1$, $\Delta \kappa_{\gamma}=\kappa_{\gamma}-1, \lambda_{Z}$ and $\lambda_{\gamma}$, which are zero in the SM [29]. In the LEP parameterisation, $\Delta \kappa_{Z}=\Delta g_{1}^{Z}-\Delta \kappa \gamma \cdot \tan ^{2} \theta_{W}$ [30]. Limits have been set by the ATLAS, CMS, CDF and D $\varnothing$ collaborations [17] [27] [19] [31]. The recent DØ results include the combination of the $\mathrm{W} \gamma, \mathrm{WW}$ and $\mathrm{WZ}$ final states to increase sensitivity. The results are compared in Figure 9. No deviation from the SM is observed. 

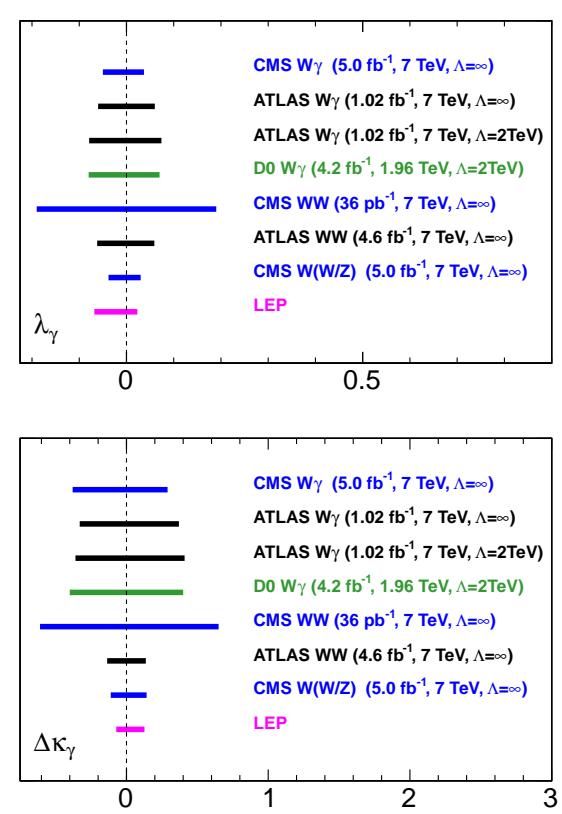

Figure 8. Summary of limits on anomalous $\mathrm{WW} \gamma$ triple gauge couplings measured in the $\mathrm{W} \gamma$ and WW final states.

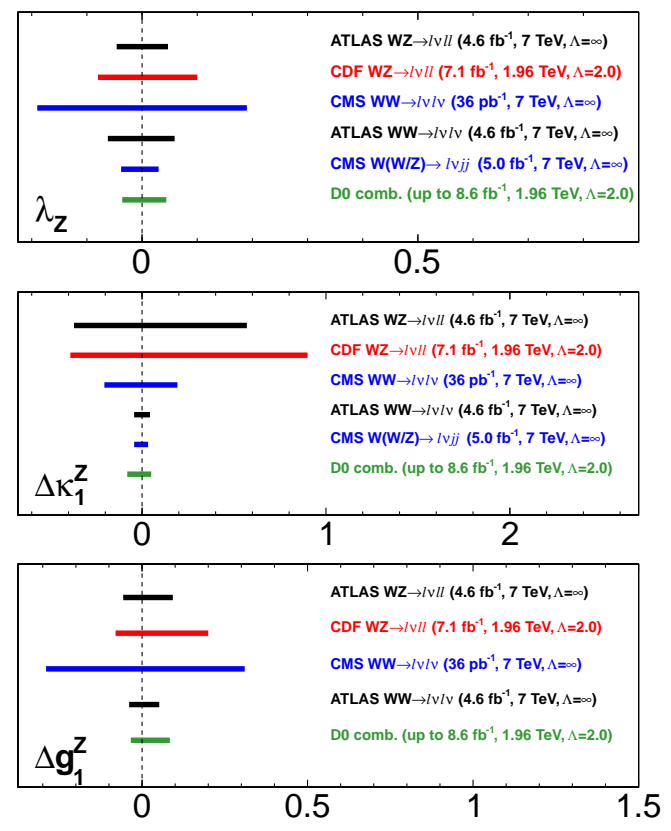

Figure 9. Summary of limits on anomalous WW $\gamma$ and WWZ triple gauge couplings measured in the $\mathrm{W} \gamma, \mathrm{WW}$ and $\mathrm{WZ}$ final states.

\subsection{Neutral couplings}

The aTGC parameters for the $\mathrm{ZV} \gamma$ vertex, where $\mathrm{V}=\mathrm{Z}$ or $\gamma$, are chosen to be $h_{3}^{\mathrm{V}}$ and $h_{4}^{\mathrm{V}}$ [32]. Limits have been set by both ATLAS and CMS, CDF and D $\varnothing$, by using the photon $p_{\mathrm{T}}$ spectrum in the $\mathrm{Z} \gamma$ final state [3] [9] [7] [8]. In new results presented at this conference, the CMS collaboration are able to improve the limit by combining the results from
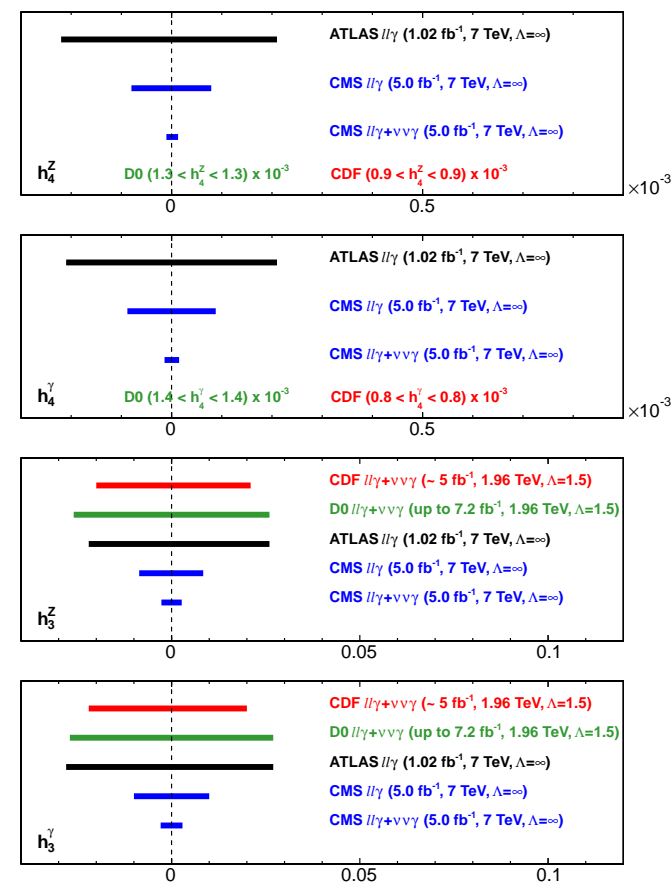

Figure 10. Summary of the limits on anomalous $\mathrm{ZZ} \gamma$ and $\mathrm{Z} \gamma \gamma$ triple gauge couplings measured in the $\mathrm{Z} \gamma$ final state.

the $\ell \ell \gamma$ and $v v \gamma$ final states. The results, which agree with SM expectations, are summarised in Figure 10.

The aTGC parameters for ZZ production are chosen to be $h_{4}^{\mathrm{V}}$ and $f_{5}^{\mathrm{V}}$, which are zero in the SM [33]. This final state is unique in providing access to the $\mathrm{ZZZ}$ coupling. No evidence of aTGC is found, so limits have been set. ATLAS has improved on its previous result [34] by a factor of around five in new results presented at this conference, by increasing the size of the dataset used, adding the dilepton $+E_{\mathrm{T}}^{\text {miss }}$ final state, and by using the $\mathrm{Z}$ boson $p_{\mathrm{T}}$ spectrum instead of performing a single counting experiment [21]. The CMS limits are set by using the fourlepton invariant mass distribution [22]. These results are compared in Figure 11.

\section{Conclusion}

Measurements of diboson production by the ATLAS, CMS, CDF and DØ experiments have been presented, covering different production modes and centre of mass energies. The measured production cross sections are typically in agreement with SM predictions, with a typical precision comparable to or better than the size of NLO corrections [13]. Events selected in these channels have been used to constrain new physics, by setting limits on anomalous triple gauge couplings. Measurements from the LHC experiments now provide the most sensitive limits in most channels. The emergence of channel combinations from both the Tevatron experiments and the LHC, continues to increase sensitivity to aTGC. 

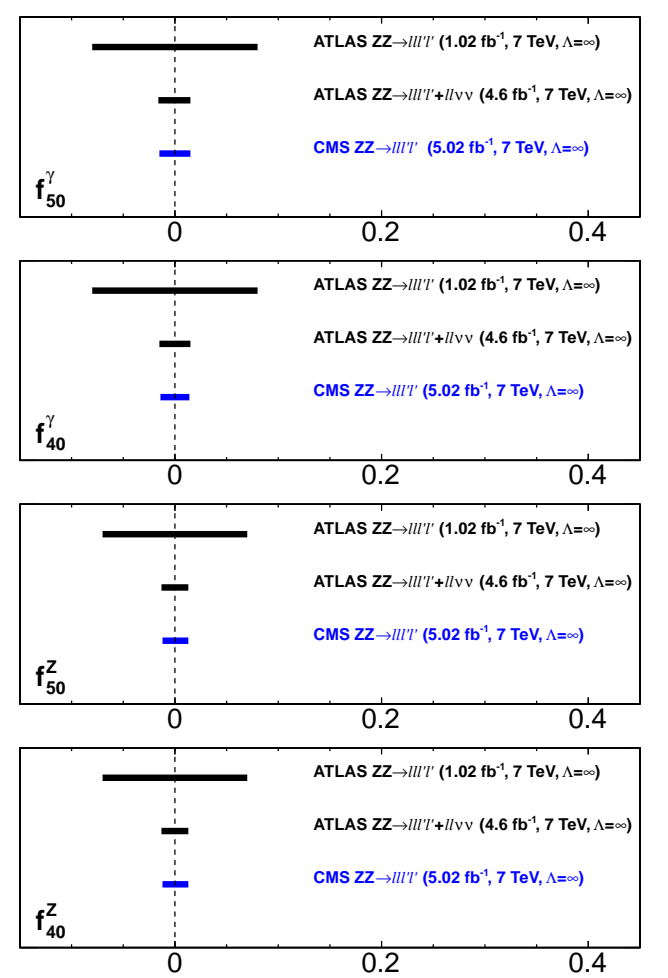

Figure 11. Summary of the limits on anomalous $\mathrm{ZZZ}$ and $\mathrm{ZZ} \gamma$ triple gauge couplings measured in the $\mathrm{Z} \gamma$ final state.

\section{Acknowledgments}

It is my pleasure to thank to the local committee for organising and hosting this interesting conference in Kyoto, and my colleagues from the ATLAS, CMS, CDF and D $\varnothing$ collaborations for their hard word in producing the results presented here.

\section{References}

[1] S. Chatrchyan et al. (CMS), Phys. Lett. B 716, 30 (2012), 1207.7235

[2] G. Aad et al. (ATLAS), Phys. Lett. B 716, 1 (2012), 1207.7214

[3] G. Aad et al. (ATLAS Collaboration), Phys.Lett. B717, 49 (2012), 1205. 2531

[4] S. Chatrchyan et al. (CMS Collaboration), CMSPAS-EWK-11-009 (2012)

[5] A. Nagano, FERMILAB-THESIS-2008-96 (2008)

[6] V.M. Abazov et al. (D0 Collaboration), Phys.Rev.Lett. 107, 241803 (2011), 1109. 4432

[7] T. Aaltonen et al. (CDF Collaboration), Phys.Rev. D82, 031103 (2010), 1004. 1140

[8] V.M. Abazov et al. (D0 Collaboration), Phys.Rev. D85, 052001 (2012), 1111. 3684
[9] S. Chatrchyan et al. (CMS Collaboration), CMSPAS-SMP-12-020 (2012)

[10] V. Abazov et al. (D0 Collaboration), Phys.Rev.Lett. 102, 201802 (2009), 0902 . 2157

[11] G. Aad et al. (ATLAS Collaboration) (2012), 1210. 2979

[12] S. Chatrchyan et al. (CMS Collaboration), CMSPAS-SMP-12-005 (2012)

[13] J.M. Campbell, R.K. Ellis, C. Williams, JHEP 07, 018 (2011), 1105.0020

[14] S. Chatrchyan et al. (CMS Collaboration) (2013), 1301.4698

[15] S. Chatrchyan et al. (CMS Collaboration) (2012), 1210.7544

[16] V.M. Abazov et al. (D0 Collaboration), Phys.Rev.Lett. 108, 181803 (2012), 1112.0536

[17] G. Aad et al. (ATLAS Collaboration), Eur.Phys.J. C72, 2173 (2012), 1208. 1390

[18] S. Chatrchyan et al. (CMS Collaboration), CMSPAS-EWK-11-010 (2011)

[19] T. Aaltonen et al. (CDF Collaboration), Phys.Rev. D86, 031104 (2012), 1202.6629

[20] V.M. Abazov et al. (D0 Collaboration), Phys.Rev. D85, 112005 (2012), 1201. 5652

[21] G. Aad et al. (ATLAS Collaboration) (2012), 1211.6096

[22] S. Chatrchyan et al. (CMS Collaboration), JHEP 1301, 063 (2013), 1211. 4890

[23] G. Aad et al. (ATLAS Collaboration), ATLASCONF-2012-090 (2012)

[24] T. Aaltonen et al. (CDF Collaboration), Phys.Rev.Lett. 108, 101801 (2012), 1112.2978

[25] S. Chatrchyan et al. (CMS Collaboration), JHEP 1212, 034 (2012), 1210. 3844

[26] U. Baur, E.L. Berger, Phys.Rev. D41, 1476 (1990)

[27] S. Chatrchyan et al. (CMS Collaboration), Phys.Lett. B699, 25 (2011), 1102 . 5429

[28] J. Alcaraz et al. (ALEPH Collaboration, DELPHI Collaboration, L3 Collaboration, OPAL Collaboration, LEP Electroweak Working Group) (2006), hep-ex/0612034

[29] K. Hagiwara, R. Peccei, D. Zeppenfeld, K. Hikasa, Nucl.Phys. B282, 253 (1987)

[30] G. Gounaris, J. Kneur, D. Zeppenfeld, Z. Ajaltouni, A. Arhrib et al. (1996), hep-ph/9601233

[31] V.M. Abazov et al. (D0 Collaboration), Phys.Lett. B718, 451 (2012), 1208. 5458

[32] U. Baur, E.L. Berger, Phys.Rev. D47, 4889 (1993)

[33] U. Baur, D.L. Rainwater, Int.J.Mod.Phys. A16S1A, 315 (2001), hep-ph/0011016

[34] G. Aad et al. (ATLAS Collaboration), Phys.Rev.Lett. 108, 041804 (2012), 1110.5016 